\title{
The change-of-standard effect: Distorted standards and adjusted impressions
}

\author{
Thomas L. Spalding And Allison C. Mullaly \\ University of Alberta, Edmonton, Alberta, Canada \\ AND \\ Victoria L. Phillips \\ Arizona State University, Tempe, Arizona
}

\begin{abstract}
Previous accounts of the memory distortion known as the change-of-standard effect hypothesize that participants form a relative impression of a target at encoding and later use that impression with the average of all items to recall the target (Higgins \& Lurie, 1983). In three experiments, we investigated the standard and the integration of the standard with the relative impression. Experiments 1 and 2 show that participants' subjective average at recall is distorted toward recent stimuli: It is computed when required and is therefore affected by the items' accessibility at that time. Furthermore, the impression's influence on recall is relatively small when the context changes between encoding and decoding. Experiment 3 shows that this change in the impression's influence occurs only when the participant integrates information across sessions, suggesting that such tasks make participants aware of the changed context and cause them to adjust the use of their impression in recalling the target.
\end{abstract}

Imagine the following scenario: You read about sentences given by Judge Jones. Jones's sentences are consistently longer than those given by other judges and, thus, strike you as harsh. Later, you read about more judges and try to recall Jones's sentences. Remembering that Jones was harsh, you think about the average of all the judges' sentences and recall longer than average sentences for Jones. If the new judges gave sentences similar to those given by the earlier judges, the recalled sentence length should be close to Jones's actual sentences. But if the new judges gave longer sentences than the first group's, you would overestimate Jones's sentence length. Similarly, if the new judges gave shorter sentences than the first group's, you would underestimate Jones's sentence length. The distortion of recall in this example is an illustration of the change-of-standard (COS) effect (Higgins \& Lurie, 1983).

The COS effect is an example of a general class of phenomena involving the processing of both item-specific and more general information. Related phenomena occur in a number of areas of cognitive psychology (see, e.g., Brown \& Siegler, 1993; Tversky \& Kahneman, 1974), as well as in social cognition (see, e.g., Higgins \& Lurie, 1983; Parducci, 1965), discrimination learning (see, e.g., Thomas, 1993), and perception and psychophysics (see, e.g., Choplin \& Hummel, 2002; Helson, 1964). Theoretical explanations and experimental paradigms differ somewhat across these areas, but there is general agree- ment on two points: (1) Individual stimuli are often encoded relative to more general information, such as an abstraction across related stimuli; and (2) such general information about related stimuli is used to decode the relative encoding of the individual stimuli into a nonrelative measure for tasks such as estimation or recall. Thus, this relative encoding-decoding cycle appears to be a fundamental aspect of performance across an extraordinarily wide range of tasks.

The COS paradigm is a particularly interesting testbed for research on this general issue, because the whole cycle is included within the paradigm, unlike some of the related areas of research, which deal with either the encoding phase or the decoding phase of the cycle only. As a result, an understanding of the cognitive mechanisms that underlie the COS effect would provide insight into this relative encoding-decoding cycle across many areas of cognition, social cognition, and perception. However, although the COS effect itself is well established (i.e., recall is distorted due to a changing standard and to a relative encoding of the to-be-recalled target), the underlying cognitive mechanisms are not yet clear. The goal of the present study was to investigate some of the underlying cognitive mechanisms that give rise to the COS effect. In particular, we investigated the processes underlying the access (or creation) of the standard at decoding and the reasoning processes underlying the combination of the relative encoding and the standard in recalling the target; the initial 
relative encoding is not a focus of the present article-for an extensive discussion of relative encoding effects, see Mussweiler (2003).

We begin by presenting the COS paradigm in somewhat more detail. In Higgins and Lurie's (1983) original study, participants were given a brief biography of one trial judge, Judge Jones, and were instructed to form an impression of him. They then read about some sentencing decisions given by Jones, along with sentencing decisions given by three unnamed judges. Participants were assigned to one of two context groups, in which the three unnamed judges gave consistently higher (high 1 ; hereafter, H1) or lower (low 1; hereafter, L1) sentences than those given by Jones. Jones's sentences were identical for all participants. After reading the information, participants rated Jones's "harshness." After a 1-week delay, the participants returned to read about the sentencing decisions given by three new, unnamed judges. These new judges gave consistently higher sentences (high 2; hereafter, H2) or lower sentences (low 2; hereafter, L2) than those given by Jones at Session 1. At this second session, there was no mention of Jones. Participants were then asked to recall Jones's sentences from the previous week.

Higgins and Lurie (1983) found that participants recalled sentences for Jones that were above (when Jones was rated as harsh) or below (when Jones was rated as lenient) the standard established by the other judges' sentences. Higgins and colleagues interpreted their data in the following way: Participants use the unbiased average ${ }^{1}$ as a standard for decoding their original impression of the target item (i.e., their belief about Jones's harshness) to reconstruct specific information about the target (i.e., Jones's sentence length). In essence, the claim is that the recalled target value is adjusted up or down from the standard, in accord with a participant's belief about Jones's degree of harshness. In other words, the effect of the impression of harshness on recall of the target is the difference between the recalled target value and the unbiased average of the context items (i.e., the effect of impression on target recall $=$ target recall - unbiased average). This interpretation depends on a participant's ability to remember his or her impression of Jones (Higgins \& Liberman, 1994; Higgins \& Lurie, 1983; Higgins \& Stangor, 1988; see also Smith, 1998) and on the accessibility of the unbiased average for use as the standard. Higgins and Liberman further concluded that the distortion in recalling the target was caused by participants' lack of understanding of the implications of relative encoding rather than by a lack of awareness of the relative nature of the encoding. These conclusions, if true, would provide a theoretical understanding of a very fundamental process. This understanding, in turn, would require cognitive mechanisms to explain the access or creation of the unbiased standard at decoding, the maintenance of the impression, and the combination of the standard and the impression, such that the combination would be consistent with participants' being aware of the relative nature of the impression but not understanding what that relative nature implies for recall.

However, if these conclusions do not hold, the required mechanisms would be quite different. We believe that all of the specific conclusions listed above are still open to question. First, in terms of the unbiased standard, Helson (1964) showed, across numerous kinds of stimuli in psychophysical studies, that the standard against which new or focal stimuli are relatively encoded depends on many characteristics of the objects or events that contribute to that standard, such as size, frequency, nearness, and intensity. Most important for the present purposes, such standards (the adaptation level, in Helson's terms) are highly sensitive to recency. Similarly, although research on intuitive statistics demonstrates that estimated means can be quite accurate under some circumstances, it also suggests that such estimated means are created by retrieving stimuli from memory (see, e.g., Malmi \& Samson, 1983), so that there could be a memory bias associated with the recency of the items that contribute to the estimated mean. In fact, much research has indicated that recent numbers have large effects on estimation (e.g., Brown, 2002; Clark, Martin, \& Henry, 1993).

Second, although participants can remember their initial impressions, this is not direct evidence that they use them equivalently across all conditions during decoding. The target-recall data in Higgins and colleagues' experiments can support this interpretation only if one assumes an unbiased standard. That is, the magnitude of the difference between the target-recall data and the unbiased standard was roughly equivalent across conditions. However, if the participants used a biased standard, the difference between the target recall and the unbiased average would not accurately measure the use of the impression at recall, because that difference would also contain the bias in the standard actually used by the participants. That is, the effect of impression on target recall actually equals target recall minus the biased average, rather than target recall minus the unbiased average. Thus, although the magnitude of the effect of the impression on recall seemed roughly equivalent across conditions in Higgins and colleagues' experiments, that rough equivalence only holds if the participants are assumed to use the unbiased averages (or at least averages in which the bias is equivalent across conditions). Therefore, whether participants use the original impression equivalently across conditions is an open question, at least until the unbiased nature of the standard is confirmed, because the conditions differ in terms of what values are recent, and therefore very possibly in terms of the degree and direction of bias in the average. Third, Higgins and Liberman's (1994) conclusion that the COS effect was caused by participants' lack of understanding of the implications of relative encoding also depends on the assumption that participants use an unbiased standard to decode their impression, and is therefore open to question.

Both the distortion of recall in the COS paradigm and the fact that the distortion is caused by the combined effects of the relative encoding and the standard are well established. As discussed above, however, exactly how these effects arise is not as clear. The goal of this article is to investigate the underlying cognitive mechanisms that give rise to the COS effect - in particular, the processes underlying the access or creation of the standard at decoding and the reasoning processes underlying the combina- 
tion of the relative encoding and the standard at decoding. In so doing, we will also investigate whether the specific conclusions drawn from the previous studies can be maintained. In particular, we will investigate what standard is used and whether the impression of harshness is used equivalently across all conditions during target recall, which in turn will have implications for the awarenessversus-understanding issue.

\section{EXPERIMENT 1}

In Experiment 1, we sought to disentangle the two contributors to the COS effect: the impression of the target (i.e., the target's relative encoding) and the standard available for decoding that impression. Experiment 1 was nearly identical to the first COS studies (Higgins \& Lurie, 1983), except that participants were asked to recall the overall average of all the items that they had seen, in addition to the target item. Our prediction was that the average would be distorted toward the most recent context items. Once the nature of the standard is established, one can investigate how recall of the target is related to the impression formed at Session 1 and the standard. In particular, the difference between the recalled target value and the estimated average gives a measure of the use of the impression in recall. If participants use their original impression in recall equivalently across conditions, then this recall difference score should be affected only by the context at the first session because the impression itself is formed only with respect to the context at the first session. If the second session context affects the recall difference score, then something about the impression or its use has changed between the creation of the impression and recall of the target.

\section{Method}

Design. Participants were randomly assigned to one of four context conditions in a $2 \times 2$ design. Each condition represented a different pairing of context information across Sessions 1 and 2. The context judges' sentences were either higher (high; hereafter, H) or lower (low; hereafter, L) than the target judge's sentences. Context (H vs. L) was crossed with Session (1 vs. 2), leading to four experimental conditions: $\mathrm{H}-\mathrm{H}, \mathrm{H}-\mathrm{L}, \mathrm{L}-\mathrm{H}$, and $\mathrm{L}-\mathrm{L}$ (see Table 1).

Participants. A total of 104 introductory psychology students participated in exchange for course credit. Ten participants failed to return for Session 2 and were removed from all analyses. In addition, participants who provided incomplete responses at Session 2 were removed prior to analysis. In total, 90 participants were used in the analyses.

Materials. Materials were modeled after those used by Higgins and Lurie (1983). At Session 1, participants received information

Table 1

Target and Context Values (in Years) in Experiment 1

\begin{tabular}{lccc}
\hline & & \multicolumn{2}{c}{$\begin{array}{c}\text { Mean Context } \\
\text { Judges' Sentences }\end{array}$} \\
\cline { 3 - 4 } & Target & $\begin{array}{c}\text { High Context } \\
\text { Judge's }\end{array}$ & $\begin{array}{c}\text { Low Context } \\
\text { (Lenient }\end{array}$ \\
Crime & Sentences & Target) & Target) \\
\hline Armed robbery & 7 & 11.0 & 3.0 \\
Fraud & 5 & 8.0 & 2.0 \\
Vandalism & 3 & 4.5 & 1.5 \\
\hline
\end{tabular}

packets containing the sentencing decisions given by each of four different trial judges. The four judges included a target judge (Judge Jones) and three unnamed judges. Each judge rendered sentences for three criminal offenses: armed robbery, fraud, and vandalism. Four specific crime scenarios, one for each trial judge, were created for each of the three offense types. In each case, the defendant was found guilty of the crime and sentenced to a jail term. Each packet contained three pages of sentencing decisions, one for each offense type. On a single page, participants saw the sentencing decisions given by the four trial judges for a single offense category, such as armed robbery.

The order of the four judges on each page was counterbalanced using a Latin square design. The order in which the offenses were presented was also counterbalanced. In the first session, the average of the sentences given by the unnamed judges was consistently higher or consistently lower than the sentences given by Judge Jones. Judge Jones's sentences were the same across all conditions. The final page of each packet contained a visual analog scale $(13 \mathrm{~cm}$ long with vertical lines as stop points at each end) used for rating Judge Jones's harshness or leniency; the left endpoint was labeled "very lenient," and the right endpoint was labeled "very harsh."

At Session 2, participants read about three new, unnamed judges. Sentencing decisions were provided for novel crimes across the three offense types. As in Session 1, the average sentences given by the unnamed judges were either consistently higher or consistently lower than Judge Jones's sentences in the first session. In Session 2, no mention was made of Judge Jones during the learning phase.

Procedure. Participants were told they were taking part in a twosession study of personal impressions and opinions. At Session 1, the instructions emphasized that participants should pay particular attention to the target item (Judge Jones). After reading all sentencing decisions, participants rated Judge Jones's harshness by marking a visual analog scale.

Participants returned 2 days later to read more information about trial judges. Participants read about sentencing decisions of three new, unnamed trial judges across three offenses. Participants were then asked to recall Judge Jones's sentences for each crime type and to recall the average sentences for each type of crime. Participants were specifically instructed to consider all the judges encountered over the two separate sessions. The order of the two tasks was counterbalanced across participants.

\section{Results and Discussion}

Four measures (see Table 2) were analyzed: (1) the target rating, (2) a target recall score, (3) an average recall score, and (4) a recall difference score (target recall - average recall). Harshness ratings for each participant were provided at Session 1, and were measured in centimeters from the scale's left endpoint. Three recall measures were calculated from Session 2. Participants recalled Judge Jones's sentencing decisions for each of the three crime types: armed robbery, fraud, and vandalism. A sentencing recall score was calculated for each participant by summing these three recalled values (following Higgins \& Lurie, 1983). Participants were also asked to recall the overall average sentencing decision for each crime type. As with target recall, average recall scores were calculated by summing recalled averages. This measure provided an estimate of the standard used to reconstruct Jones's sentencing decisions. Finally, a recall difference measure was calculated by subtracting the average recall score from its target recall counterpart. The recall difference scores indicated whether the target was recalled as being higher than, lower than, or the same as the perceived standard. This measure was used to isolate the effect of each partici- 
Table 2

Mean ( $S E$ ) Recalled Sentences for Accurate Average, Judge Jones, the Recalled Average, and the Recall Difference for Experiment 1

\begin{tabular}{|c|c|c|c|c|c|c|c|c|c|}
\hline \multirow[b]{2}{*}{ Context 1} & \multirow[b]{2}{*}{ Context 2} & \multirow[b]{2}{*}{$n$} & \multirow{2}{*}{$\begin{array}{l}\text { Accurate } \\
\text { Average }\end{array}$} & \multicolumn{2}{|c|}{$\begin{array}{c}\text { Recall: } \\
\text { Judge Jones }\end{array}$} & \multicolumn{2}{|c|}{$\begin{array}{l}\text { Recall: } \\
\text { Average }\end{array}$} & \multicolumn{2}{|c|}{$\begin{array}{c}\text { Recall } \\
\text { Difference }\end{array}$} \\
\hline & & & & $M$ & $\overline{S E}$ & $M$ & $\overline{S E}$ & $M$ & $\overline{S E}$ \\
\hline \multirow[t]{2}{*}{ High $1^{*}$} & High 2 & 24 & 22.4 & 21.0 & 1.3 & 22.6 & 0.7 & -1.6 & 1.1 \\
\hline & Low 2 & 21 & 15.0 & 11.8 & 1.3 & 10.9 & 0.7 & 0.9 & 1.1 \\
\hline \multirow[t]{2}{*}{ Low $1^{* *}$} & High 2 & 22 & 15.0 & 24.0 & 1.3 & 22.2 & 0.7 & 1.8 & 1.1 \\
\hline & Low 2 & 23 & 7.7 & 12.3 & 1.3 & 7.4 & 0.7 & 4.9 & 1.1 \\
\hline
\end{tabular}

${ }^{*}$ Mean harshness rating at Session $1=5.13 .{ }^{* *}$ Mean harshness rating at Session $1=9.30$.

pant's belief about Jones's harshness from the effect of the standard in recall of the target. Thus, the recall-difference score measures the use of the impression of harshness in target recall.

Judgment of the target. Harshness ratings were significantly different as a function of context at Session 1 $\left[F(1,86)=83.2, M S_{\mathrm{e}}=4.7, p<.05\right.$; all other $\left.F_{\mathrm{s}}<1\right]$. As expected, Judge Jones was rated as harsh in the L1 context $(M=9.3, S E=0.4)$ and lenient in the H1 context $(M=$ $5.1, S E=0.3)$. Not surprisingly, the impression of Jones depended only on the context in which he was encountered and was unaffected by the Session 2 context manipulation. Thus, if the recall-difference score analyzed below was affected by the context at Session 2, that effect cannot be attributed to, for example, a spurious difference in the judged harshness of the target across Session 2's context conditions.

Recall of the target. Although the actual sentences rendered by Jones were the same in all conditions, recall of Judge Jones's sentences was reliably different as a function of context at Session $2\left[F(1,86)=65.0, M S_{\mathrm{e}}=38.0\right.$, $p<.05]$, with no significant effect of context at Session 1 $\left[F(1,86)=1.8, M S_{\mathrm{e}}=38.0, p>.05\right]$ and no interaction $(F<1)$. Recalled sentences were higher when sentencing values were high at Session $2(M=22.5, S E=1.0)$ and, likewise, recalled sentences were lower when sentencing values were low at Session $2(M=12.1, S E=0.8)$. The lack of effect of Session 1 is likely due to the fact that Session 1 had two opposite effects on target recall (i.e., high numbers for context judges at Session 1 should have led to a higher average, but also to the impression that Jones gives low sentences). In general, the recalled sentences for Jones closely parallel those found in Higgins and Lurie (1983).

Recall of the overall average. For average sentence recall, both main and interactive effects of context at Sessions 1 and 2 were found. Recall was higher given high recent context values (for $\mathrm{H} 2, M=22.4, S E=0.4$ ) and lower given low recent context values (for L2, $M=9.1$, $S E=0.6)\left[F(1,86)=382.8, M S_{\mathrm{e}}=10.3, p<.05\right]$. Context at Session 1 also influenced average recall, such that recall was higher when the sentencing values at Session 1 were high $(M=17.2, S E=1.0)$ than when the sentencing values were low $(M=14.6, S E=1.2)[F(1,86)=8.5$, $\left.M S_{\mathrm{e}}=10.3, p<.05\right]$. There was a significant interaction $\left[F(1,86)=5.5, M S_{\mathrm{e}}=10.3, p<.05\right]$. Simple effects tests revealed that the first session context exerted its influence on recall of the average only when the context at Session 2 was low (this interaction was not replicated in later stud- ies). Most important, the $\mathrm{L}-\mathrm{H}$ and $\mathrm{H}-\mathrm{L}$ conditions (where the true mean is 15) show a strong effect of recency (for $\mathrm{L}-\mathrm{H}, M=22.2$; for $\mathrm{H}-\mathrm{L}, M=10.9$ ). Thus, the standard at the time of recall appears to be highly biased toward the most recent items.

Recall difference: Target recall minus average recall. The preceding analyses show that recent context influenced memory of both the target and average. In order to assess the effect of the impression on target recall over and above the influence of recent information, we examined the difference between the recalled average and the recalled target sentencing behaviors. If the Session 1 context was high, the difference score should be negative because Jones was considered lenient, and if the Session 1 context was low, the difference score should be positive because Jones was considered harsh. This was the pattern found in previous research when the target recall was compared to the accurate average. Indeed, comparing the target recall to the accurate average would give much the same result for the present data: The differences between target recall and the accurate standard for each condition in the present study $(\mathrm{H}-\mathrm{H},-1.3 ; \mathrm{H}-\mathrm{L},-3.2 ; \mathrm{L}-\mathrm{H}, 9.0$; $\mathrm{L}-\mathrm{L}, 4.6)$ were similar to the values Higgins and Lurie (1983) obtained (H-H, - 1.3; H-L, -3.7; L-H, 2.9; L-L, 4.6). According to this view, the impression from Session 1 (along with the standard) is what participants use to recall Jones's sentences. Session 2 should not affect the impression formed at Session 1 and used at recall.

However, when target recall and the estimated average are used to create the recall-difference scores, a different pattern emerges. The expected main effect of context at Session 1 was obtained $\left[F(1,86)=11.8, M S_{\mathrm{e}}=26.5\right.$, $p<.05]$. In the $\mathrm{H} 1$ (lenient judgment) condition, recalled sentences for Jones were lower than the subjective average $(M=-0.4, S E=0.7)$. By contrast, in the L1 (harsh judgment) condition, recalled sentences for Jones were higher than the subjective average $(M=3.4, S E=0.8)$. However, the recall-difference score was also affected by Session 2's context $\left[F(1,86)=6.6, M S_{\mathrm{e}}=26.5, p<.05\right]$. There was no interaction $(F<1)$.

These main effects are clarified in Figure 1. Consider the two conditions where Jones was harsh (L1). When the context did not change (L-L), Jones's sentences were nearly 5 years higher than the average. By contrast, when the context changed $(\mathrm{L}-\mathrm{H})$, Jones's sentences exceeded the average, but by less than 2 years. Similarly, when Jones was lenient $(\mathrm{H} 1)$ and the context did not change $(\mathrm{H}-\mathrm{H})$, Jones's sentences were lower than the average by 1.6 years. 


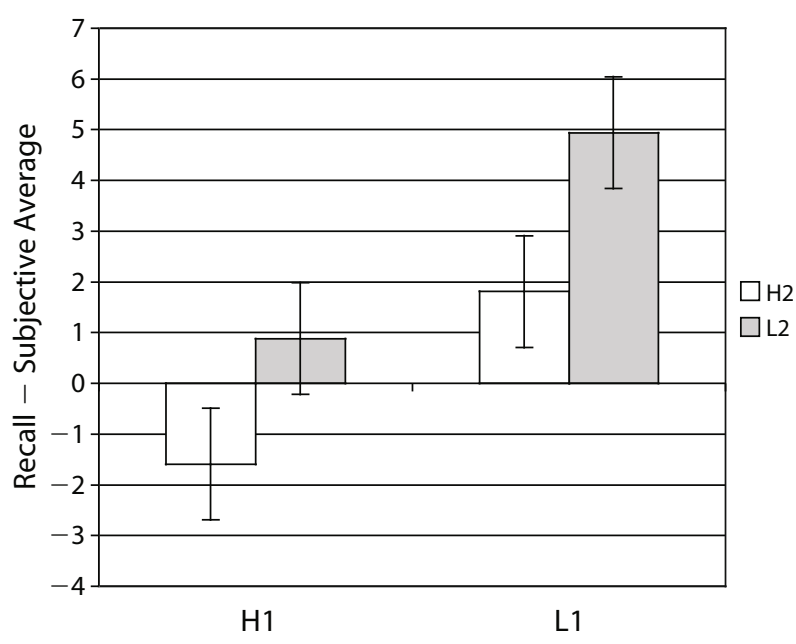

Figure 1. The impression effect (recall - subjective average) in Experiment 1, plotted by the following conditions: H1 (high numbers in Session 1); L1 (low numbers in Session 1); H2 (high numbers in Session 2); and L2 (low numbers in Session 2). Error bars represent $S E$ s.

However, when the context changed $(\mathrm{H}-\mathrm{L})$, participants recalled that Jones's sentences exceeded the average by 0.9 years, even though he was lenient. It is important to note that, when the context changed from Session 1 to Session 2, the recall-difference score was quite small (in absolute value); but when the context did not change, the recall-difference score was relatively large. Thus, recall is not a simple, consistent, or equivalent combination of the original impression with the current (distorted) standard across the conditions. Rather, it seems that the degree to which the impression of the target affects target recall is related to whether the context changes from Session 1 to Session 2. Note that this view of the influence of the impression on recall is very different from that suggested by comparing recall to the accurate average, as was done above (and in previous research).

Replications and posttest. We conducted a full replication with a different material set (home electronics prices) and again found that the subjective average was distorted toward the recent items and that the influence of the impression at decoding (as measured in the recalldifference scores) was small in the $\mathrm{L}-\mathrm{H}$ and $\mathrm{H}-\mathrm{L}$ conditions, as compared with the $\mathrm{L}-\mathrm{L}$ and $\mathrm{H}-\mathrm{H}$ conditions. We again replicated (using both material sets) just the $\mathrm{H}-\mathrm{L}$ and $\mathrm{L}-\mathrm{H}$ conditions. Again, in both material sets, we found a strong distortion of the subjective average toward the recent context items but only quite small effects of the impression in the recall-difference score.

One possible explanation for the small influence of the impression in recalling the target in the $\mathrm{L}-\mathrm{H}$ and $\mathrm{H}-\mathrm{L}$ conditions is that, in these conditions, large impression effects would lead to implausible sentence values. Consider the $\mathrm{L}-\mathrm{H}$ condition: The subjective average sentence is already quite long, and participants think Jones should give longer than average sentences. Perhaps the recall-difference score is small because the sentences begin to seem implausible, according to the participants' prior knowledge (i.e., even though Jones is harsh, an average sentence of 10 years for armed robbery seems high, so participants are unwilling to recall a much longer sentence). We conducted an additional experiment using fictional materials, ensuring that participants had no prior expectations of plausible values (J-ratings measured in picoFarads described electrical components). The results were identical to those in Experiment 1: a distorted subjective average and the same pattern of recall-difference scores (see Figure 2). Thus, the pattern obtained in Experiment 1 is unlikely to be due to prior knowledge of plausible values.

Regression analyses. Although it is clear that participants' recalled averages are distorted toward the most recent context, this does not constitute direct evidence that these distorted averages are used in recalling the target. It is possible, though perhaps unlikely, that the participants have access to the unbiased averages in recalling the target, but not when asked directly for the average. In order to provide some evidence that the distorted averages are used in recalling the target, we performed a series of regression analyses in which we predicted the recalled target values using the unbiased average, the distorted (recalled) average, and the rating of harshness from Session 1 . A model using only the unbiased average accounts for $17.8 \%$ of the variance in the target-recall data. Adding the harshness rating increases the variance accounted for by $20.3 \%$ to $38.1 \%[F(1,87)=28.5, p<.001]$. However, a model using only the distorted (recalled) average accounts for $55.5 \%$ of the variance in the target-recall data. Adding the harshness rating to this model increases the variance accounted for by $6.4 \%$ to a total of $61.9 \%$ of the variance $[F(1,87)=5.3, p<.001]$. When the unbiased average, distorted average, and harshness rating are all entered into the same model, the unbiased average is not a significant predictor $(p=.57)$. In short, the recalled average, although it is distorted toward the most recent

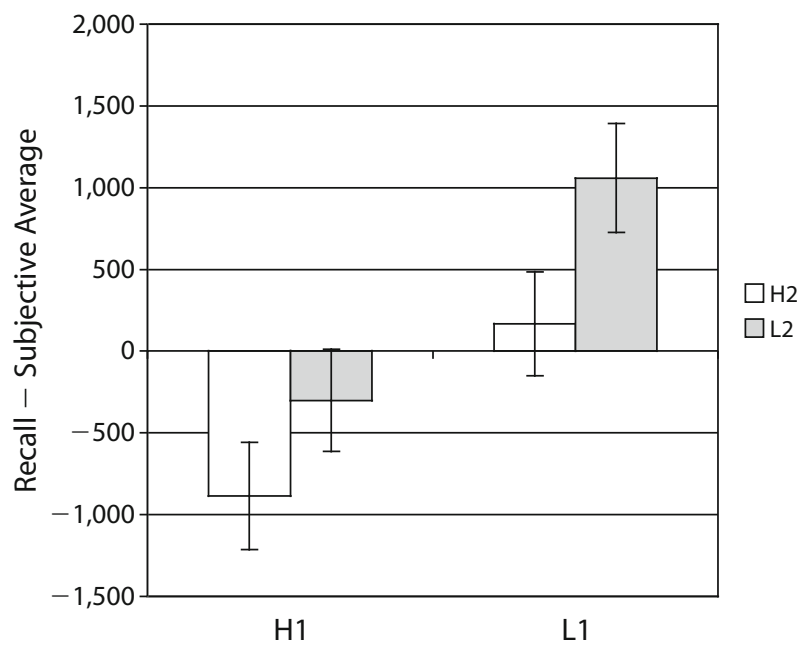

Figure 2. The impression effect (recall - subjective average) in Experiment 1 (posttest), plotted by the following conditions: H1 (high numbers in Session 1); L1 (low numbers in Session 1); H2 (high numbers in Session 2); and L2 (low numbers in Session 2).

Error bars represent $S E$ s. 
context, is a much better predictor of target recall than the unbiased average is, strongly suggesting that the distorted average is the standard that participants use to recall the target. In addition, as we suggested above, when one assumes that the unbiased average is used, the effect of the impression of harshness appears bigger than it really is, because some (but not all) of the variability associated with the bias in the average used by the participants can then be accounted for by the harshness rating.

Summary of Experiment 1. In a very general sense, our results are in accord with Higgins and Lurie's (1983) original COS effect. At encoding, the target was evaluated with respect to the context, and, at decoding, the impression of the target was used along with the current standard to recall specific target values. With respect to Higgins and colleagues' specific conclusions, however, our results are not in accord. First, as we predicted, the standard used at decoding to reconstruct the target's original behavior was heavily distorted toward the recent context. Second, the impression was less influential in driving the reconstruction of the target when the context changed, as compared with consistent contexts. The next two experiments investigate these effects in more depth. Experiment 2 investigates the distortion of the standard. Experiment 3 investigates the conditions under which the use of the impression in recall is affected by Session 2's context.

\section{EXPERIMENT 2}

Experiment 2 investigates two issues regarding the standard. First, is the distortion an artifact of the judgment task being performed at Session 1? Perhaps, in Session 1, the evaluation of the target consumed much of the participants' attention and the remaining resources were too limited to properly encode the context items. If this were the case, it would indicate that the distortion of the standard would be unlikely to extend to instances of the encoding-decoding cycle in which the standard already exists when the target is encoded. In Experiment 2, we do not present a target item. If the distortion found previously resulted from poor encoding of Session 1 items, then Experiment 2 should show no distortion.

Assuming that the impression formation task is not responsible for the distortion, is the distortion toward the recent items a recency effect or an order effect? An example of an order-based, but not recency-based, process that would lead to an average distorted toward the Session 2 items might be helpful here. Imagine that, as each item is encountered, it is averaged with the existing average. Thus, the first two items are averaged together and are equally weighted, so that each accounts for $50 \%$ of the average. The third item is averaged with the stored average, so that the first and second item each now accounts for $25 \%$ of the average and the third accounts for $50 \%$, and so on. The average is distorted toward the later items, but not as a result, strictly speaking, of those items' recency. A recency effect implies that the average is computed when needed, whereas an order effect would be compatible with an ongoing automatic computation (e.g., a running average of the type described above). Thus, the nature of the standard implies different underlying cognitive mechanisms.

We separate these possibilities by imposing a delay between the presentation of the Session 2 items and the average-estimation task. If participants compute the average when needed and the computation is affected by the relative recency of different items, the delay should reduce or eliminate the distortion in the recalled average. A delay should cause the activation of the recent items to disperse, so that the recent items would not have an undue effect on the average at the time of test. This reasoning follows research on serial position effects (e.g., Glanzer \& Cunitz, 1966), in which activation of an item decreases rapidly at first, and then more slowly later. On the other hand, if the distortion is due to order, but not recency, then a delay should have no effect. As no new item is encountered during the delay, such an order effect would be maintained across the delay.

\section{Method}

Design. Materials were prices of foods offered by various catering companies (see Table 3). Only context items were presented: There was no target item and no instructions to pay special attention to any individual item. Sessions 1 and 2 were separated by an unrelated experiment lasting approximately $25 \mathrm{~min}$. Only $\mathrm{L}-\mathrm{H}$ and $\mathrm{H}-\mathrm{L}$ conditions were included; distortion of the average can be detected only in these conditions. The true average of the items in these two conditions was identical. The second factor was whether the average-estimation task was given immediately or after another delay (filled with a reading task). Thus, the experiment was a $2 \times 2$ design with order ( $\mathrm{L}-\mathrm{H}$ vs. $\mathrm{H}-\mathrm{L}$ ) as one factor and delay (immediate vs. delayed) as the other.

Participants. A total of 41 introductory psychology students participated in exchange for course credit.

Procedure. Participants were told that they were taking part in an experiment on impression formation and that they would be asked to read some information about a set of caterers and the prices they charged for certain foods. At Session 1, the participants saw a set of three caterers, each of which offered three kinds of foods. The materials were a direct analog to those in Experiment 1, with prices taking the place of sentences and food types taking the place of crime types. Participants then took part in an unrelated 25-min experiment. After the delay, the participants were asked to read about more caterers who offered the same types of foods. At the end of Session 2, half of the participants were asked to recall the average of all the prices that they had seen that day, separately for the three types of food. The other half of the participants engaged in another task involving reading and summarizing a short article of unrelated material (this task required about $15 \mathrm{~min}$ ). After this task, the delayed-test group completed the same average-recall task.

\section{Results and Discussion}

The recalled averages were summed across the three types of food. The data were analyzed using a $2 \times 2$ ANOVA. There was a main effect of order $[F(1,37)=$

Table 3

Target and Context Values (in Dollars) in Experiments 2 and 3

\begin{tabular}{lccc}
\hline & & \multicolumn{2}{c}{ Mean Prices } \\
\cline { 3 - 4 } Food Type & Target Price & $\begin{array}{c}\text { High Context } \\
\text { (Inexpensive } \\
\text { Target) }\end{array}$ & $\begin{array}{c}\text { Low Context } \\
\text { (Expensive } \\
\text { Target) }\end{array}$ \\
\hline Caviar & 64 & 84 & 44 \\
Cheese & 50 & 62 & 38 \\
Bread & 20 & 28 & 12 \\
\hline
\end{tabular}


9.0, $\left.M S_{\mathrm{e}}=411, p<.05\right]$ and no main effect of delay $[F(1,37)<1]$. Most important, there was a significant interaction $\left[F(1,37)=6.2, M S_{\mathrm{e}}=411, p<.05\right]$. With the immediate test, there was a large effect of order, with the $\mathrm{L}-\mathrm{H}$ condition $(M=133.4, S E=6.4)$ leading to higher recalled averages than the $\mathrm{H}-\mathrm{L}$ condition $(M=98.6, S E=$ 6.1) $\left[F(1,19)=21.1, M S_{\mathrm{e}}=301, p<.05\right]$. However, in the delayed-test condition, the $\mathrm{L}-\mathrm{H}$ condition $(M=112.3$, $S E=6.4)$ was nearly identical to the $\mathrm{H}-\mathrm{L}$ condition $(M=$ 109.1, $S E=6.4)[F(1,18)<1]$.

The distortion of the subjective average in the previous studies was not an artifact caused by the presence of the target item. This is indicated by the large distortion in the immediate-test condition. More important, the fact that there was no distortion in the delayed condition strongly suggests that the subjective average is computed when needed, rather than being automatically computed when the items are encountered, and that the accessibility of the individual items for that computation depends on their recency. In short, it appears that the standard is created using a process that relies on the retrieval of stimuli from memory, rather than on a process that relies on an ongoing abstraction process. These results provide an interesting insight into the mechanisms that underlie the creation of standards and suggest that the mechanisms that are here involved in creating standards are likely to be similar to those that are involved in creating the representations that give rise to prototype effects in category learning (for a discussion, see Malmi \& Samson, 1983; Murphy, 2002).

\section{EXPERIMENT 3}

Experiment 3 investigated the effect of the Session 2 context on the participants' use of their impression of the target item on target recall (i.e., the recall-difference score) that was found in Experiment 1. In Experiment 1, the effect of the impression was not consistent across conditions. Instead, it appears that the influence of participants' impression on target recall differed depending on whether the context in Session 2 was consistent with that in Session 1.

This experiment used a new paradigm: After the presentation of the Session 2 context items, participants were given the average cost of all of the caterers for new types of foods and were asked to estimate prices for the target catering company for those foods. The extent to which the estimated prices differed from the average price given to the participants should have been a function of the impression of the target company (i.e., the extent to which the participant believed the caterer to be expensive). Because (1) participants in all conditions received the same average price information about the new food types, (2) the averages were available throughout the estimation task, and (3) the prices of the new food types were quite different from those of the original food types, it is highly unlikely that the original prices would have affected these estimate-difference scores, except through the impression of the target caterer.

Experiment 3A replicated the Experiment 1 tasks (recalling the average price of all caterers and the target caterer price prior to the new estimation task). If the estimation task shows the same pattern of effects in the estimatedifference scores that we found in the recall-difference score in the previous experiments (i.e., relatively small difference scores when the context changes), it will be converging evidence that the effects in the previous experiments resulted from participants' recalling the target using their impression of the target item and the subjective average. In addition, it would strongly suggest that some aspect of the use of the impression is adjusted depending on the change or consistency of the contexts across sessions. If the use of the impression is not adjusted in some way during Session 2 or during the recall tasks, then only the context at Session 1 should affect the estimatedifference scores. We return to the precise nature of this adjustment in the General Discussion.

Experiments 3B, 3C, and 3D were designed to investigate at what point the adjustment is triggered. Perhaps participants immediately notice that the prices have changed at Session 2 and therefore update their impression of the target caterer prior to engaging in any recall task. On the other hand, perhaps they do not immediately notice the price changes and update their impression only when they average all the items or when they estimate the target item. The three additional experiments use only the target recall (Experiment 3B), only the average recall (Experiment 3C), or neither (Experiment 3D) between Session 2 and the estimation task. These experiments allow us to see whether the adjustment in the use of the impression is due to these tasks or to the presentation of the Session 2 items, per se.

\section{Method}

The procedures of Experiment 3 were adapted from Experiment 1. The materials were adapted from Experiment 2, with the addition of a target caterer at Session 1 whose prices were the same moderate value across all conditions. As in Experiment 2, Sessions 1 and 2 were separated by an unrelated experiment lasting approximately $25 \mathrm{~min}$. Context condition at Session 1 was crossed with context condition at Session 2, resulting in four conditions: $\mathrm{H}-\mathrm{H}, \mathrm{H}-\mathrm{L}, \mathrm{L}-\mathrm{H}$, and $\mathrm{L}-\mathrm{L}$.

Participants. A total of 309 introductory psychology students participated in exchange for course credit: In Experiment 3A, $N=$ 94; in Experiment $3 \mathrm{~B}, N=66$; in Experiment $3 \mathrm{C}, N=63$; and in Experiment $3 \mathrm{D}, N=86$. Two participants who provided estimates more than 5 standard deviations from the mean were replaced in the analysis of Experiment 3B.

Procedure. The procedure was very similar to that in Experiment 1 but included the filled delay from Experiment 2, rather than a 2-day delay. In addition, adjustments were made to the instructions due to the differences in materials. After Session 2, the participants performed the average recall and the target recall (Experiment $3 \mathrm{~A}$ ), only the target recall (Experiment $3 \mathrm{~B}$ ), only the average recall (Experiment 3C), or neither recall task (Experiment 3D). Participants then performed the estimation task: They were presented with three new types of food, along with the average price that other caterers charge for each food type, and were asked how much they thought the target caterer would charge for each kind of food.

\section{Results and Discussion}

Experiment 3A: Recall target and average. The judgment effect at Session 1 showed a contrast to the context items, such that low context prices led to an expensive 
judgment and high context prices led to an inexpensive judgment. This is true of each of the remaining experiments. The recalled average was distorted toward the most recent items, and the recall-difference scores were smaller in the $\mathrm{H}-\mathrm{L}$ and $\mathrm{L}-\mathrm{H}$ conditions than in the $\mathrm{L}-\mathrm{L}$ and $\mathrm{H}-\mathrm{H}$ conditions, as was previously found.

The critical data for these experiments are the estimatedifference scores. The estimate-difference scores were created by summing differences between the participants' estimates and the averages they were given across the food types. The estimate-difference scores (see Figure 3) were analyzed using a $2 \times 2$ ANOVA. This analysis is analogous to the recall-difference analysis from Experiment 1 and will also be used in Experiments 3B, 3C, and 3D. As in the previous experiments, there was a strong effect of context at Sessions $1\left[F(1,91)=47.8, M S_{\mathrm{e}}=707, p<.05\right]$ and 2 $\left[F(1,91)=8.8, M S_{\mathrm{e}}=707, p<.05\right]$, but no interaction.

The similarity of these results to those of Experiment 1 is quite striking. This similarity suggests three things. First, participants perform the estimation task in much the same way as they do the target-recall task. Second, participants perform both of these tasks by decoding the impression of the target with respect to a standard and, specifically, with respect to the biased (recalled) average in Experiment 1. Third, the impression formed during Session 1 is used differently at decoding, depending on whether the context is consistent across sessions, indicating that something regarding the impression has changed during the presentation of Session 2 items or during the average-recall or target-recall task.

Experiment 3B: Recall target only. The recall of the target was essentially identical to that in Experiment 3A. The estimate-difference scores are shown in Figure 4. As in the previous experiments, there was a strong effect of context at Sessions $1\left[F(1,60)=70.2, M S_{\mathrm{e}}=600, p<\right.$ $.05]$ and $2\left[F(1,60)=6.0, M S_{\mathrm{e}}=600, p<.05\right]$, but no interaction. This was more or less expected because the only difference from Experiment $3 \mathrm{~A}$ was that the participants in this experiment were not explicitly asked to recall the average. However, when recalling the target, participants were assumed to compute the average. At any rate, the adjustment to the use of the impression did not require an explicit recall of the average.

Experiment 3C: Recall average only. The recall of the average was distorted, as in the previous experiments. The estimate-difference scores are shown in Figure 5. As in the previous experiments, there was a strong effect of context at Sessions $1\left[F(1,59)=44.5, M S_{\mathrm{e}}=727, p<\right.$ $.05]$ and $2\left[F(1,59)=8.1, M S_{\mathrm{e}}=727, p<.05\right]$, but no interaction. The recall of the target item was not required for participants to adjust the use of their impression. Simply computing the average was enough to trigger the adjustment.

Experiment 3D: No task. There was no recall task prior to the estimation task in Experiment 3D. The estimate-difference scores are shown in Figure 6. As in the previous experiments, there was a strong effect of context at Session $1\left[F(1,83)=55.9, M S_{\mathrm{e}}=917, p<.05\right]$. However, there was no effect of context at Session 2 and no interaction (both $F \mathrm{~s}<1$ ). This result is clearly differ-

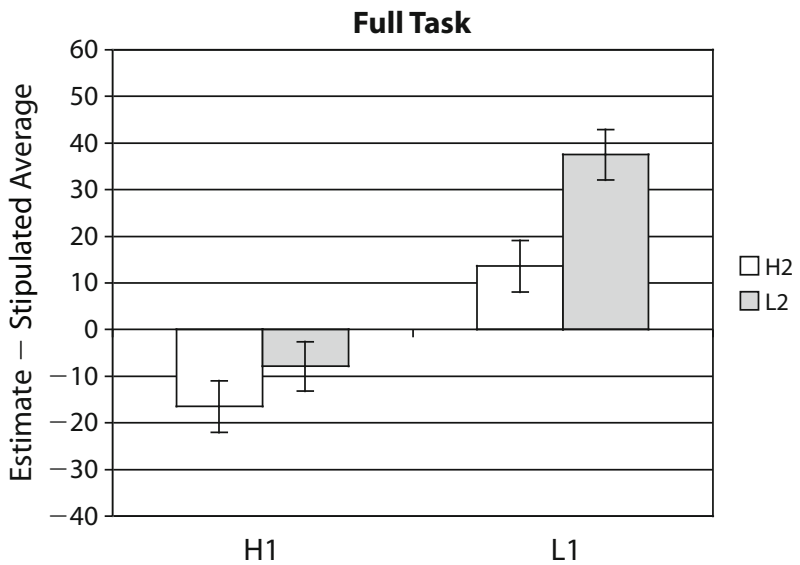

Figure 3. The impression effect (estimate - stipulated average) in Experiment 3A, plotted by the following conditions: H1 (high numbers in Session 1); L1 (low numbers in Session 1); H2 (high numbers in Session 2); and L2 (low numbers in Session 2). Error bars represent $S E$ s.

ent from those of Experiments 3A, 3B, and 3C, as well as from those of Experiment 1 and the Experiment 1 posttests. Presentation of the Session 2 items themselves was not sufficient for participants to adjust the use of their impressions (nor was the final estimation task). Thus, the adjustment appears to be triggered by some aspect of recalling the average (or the target, although this is difficult to establish as, by hypothesis, the target-recall task requires an implicit computation of the average).

Combined analysis. Given that the main difference between Experiments 3A through 3C and Experiment 3D is the lack of a significant Session 2 context effect, it would be helpful to have statistical evidence that Experiment 3D differs significantly from the rest of Experiment 3 . To this end, the estimate-difference-score data from all of Experiment 3 were combined and submitted to a three-way ANOVA with context at Session 1, context at Session 2,

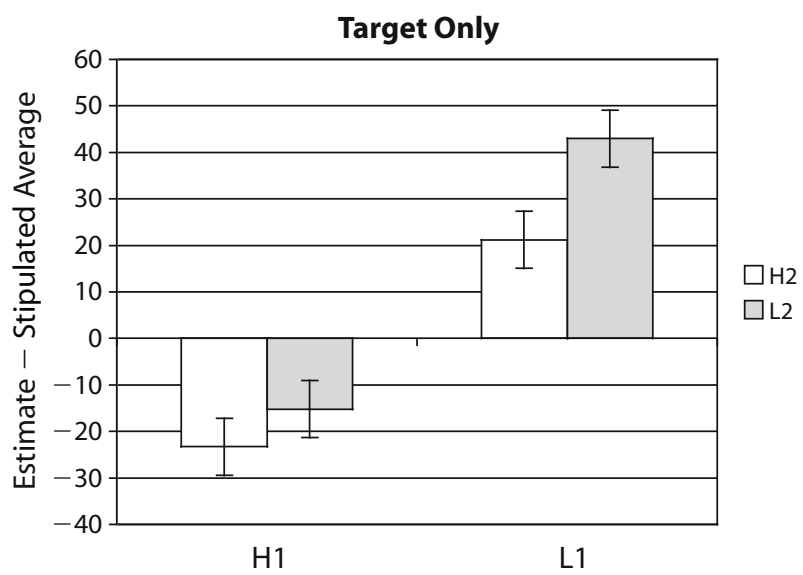

Figure 4. The impression effect (estimate - stipulated average) in Experiment 3B, plotted by the following conditions: H1 (high numbers in Session 1); L1 (low numbers in Session 1); H2 (high numbers in Session 2); and L2 (low numbers in Session 2). Error bars represent $S E$ s. 
Average Only

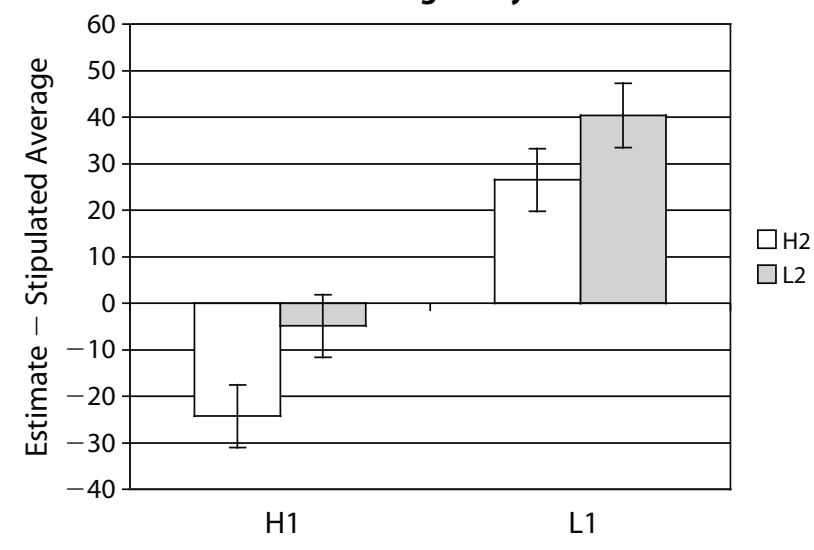

Figure 5. The impression effect (estimate - stipulated average) in Experiment 3C, plotted by the following conditions: H1 (high numbers in Session 1); L1 (low numbers in Session 1); H2 (high numbers in Session 2); and L2 (low numbers in Session 2). Error bars represent $S E$ s.

and experiment as factors. In this analysis, there was a significant Session $2 \times$ experiment interaction $[F(3,299)=$ $\left.3.0, M S_{\mathrm{e}}=764.1, p<.05\right]$. There were also significant main effects of Session $1\left[F(1,299)=215.4, M S_{\mathrm{e}}=764.1\right.$, $p<.05]$, Session $2\left[F(1,299)=11.5, M S_{\mathrm{e}}=764.1, p<\right.$ $.05]$, and experiment $\left[F(3,299)=4.7, M S_{\mathrm{e}}=764.1, p<\right.$ $.05]$. Post hoc tests on the main effect of experiment indicated that Experiment 3D differed from each of the others by Tukey's LSD (all $p$ s $<.05$ ). None of the other experiments differed significantly from each other.

Summary. Because all of the participants were given the same averages for the new products, the estimatedifference score should have been a relatively direct reflection of the impression of the target used in estimation. Across Experiments 3A, 3B, and 3C, the use of the impression was such that participants estimated prices closer to the average in the $\mathrm{L}-\mathrm{H}$ and $\mathrm{H}-\mathrm{L}$ conditions than in the

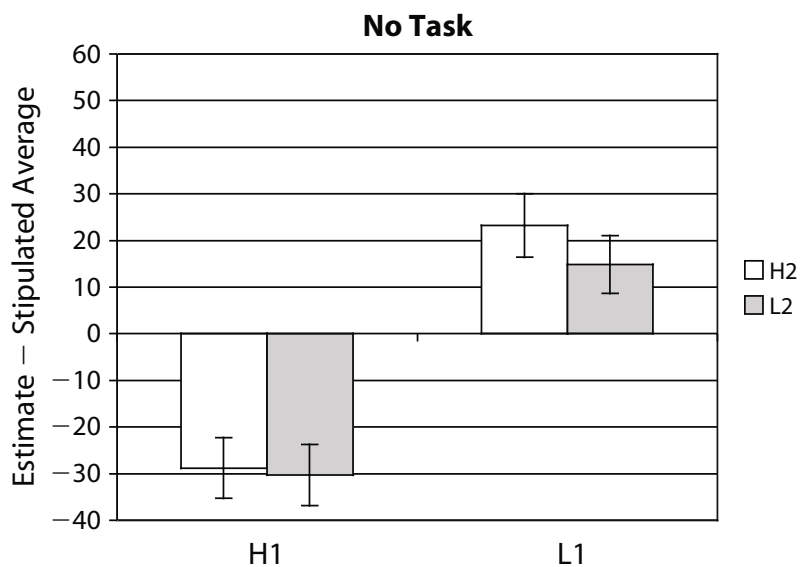

Figure 6. The impression effect (estimate - stipulated average) in Experiment 3D, plotted by the following conditions: H1 (high numbers in Session 1); L1 (low numbers in Session 1); H2 (high numbers in Session 2); and L2 (low numbers in Session 2). Error bars represent $S E$ s.
$\mathrm{H}-\mathrm{H}$ and $\mathrm{L}-\mathrm{L}$ conditions. Thus, as in Experiment 1, the effect of the impression on estimation seems to have been reduced in the conditions where the context changed or, alternatively, to be increased when the context was consistent. However, when there was no task between the presentation of Session 2 items and the estimation task, only the context at Session 1 affected the estimates, suggesting that the original impression was used in an equivalent way across conditions. It appears that the use of the impression was adjusted only when the intervening task(s) required the use of information from across the two sessions. Perhaps such tasks draw attention to the change (or continuity) in the context items. Other tasks, such as explicitly asking the participants to think back to Session 1, might cause similar effects (e.g., the nonsalient reinstatement condition in Experiment 1 of Higgins \& Liberman, 1994).

\section{GENERAL DISCUSSION}

The distorted recall of the target item in the COS paradigm was due to a changed standard from encoding to decoding and to the combination of the participants' impression of the target with that changed standard. In this very general sense, our results are consistent with those from previous research on COS effects (Higgins \& Liberman, 1994; Higgins \& Lurie, 1983; Higgins \& Stangor, 1988). However, there are important new findings with respect to the standard and to the way the impression and standard are combined in recall. In this section, we focus on these new findings and their implications for the cognitive mechanisms underlying the COS effect, as well as on their implications for participants' awareness and understanding of relative encoding. We also make brief connections to research on other examples of the relative encodingdecoding cycle.

\section{Creation of the Standard}

The standard at decoding is distorted toward the recent items. Furthermore, Experiment 2 strongly suggests that participants do not create a representation of the average of the items until it is required. This, in turn, implies that the content of the standard (and, thus, part of the distortion in recall of the target) is a function of the accessibility of the individual items at recall, which opens the door not only to recency effects but also to many kinds of effects that change the accessibility of particular items. Given that this effect depends largely on basic memory processes, it is likely to hold across many different areas that involve relative encoding-decoding cycles. Mussweiler (2003) similarly proposed that the process of comparing a target to a relevant standard in social comparisons is guided by an accessibility mechanism that selectively recruits information for comparison, so that some information is more accessible than other information.

Adaptation level theory (Helson, 1964) attempts to explain the relative encoding and decoding of perceptual stimuli-particularly in psychophysical tasks. The gist of the theory is that the perceptual system adapts its responses to the presentation of prior stimuli, so that the encoding (or decoding) of a focal stimulus is a function of 
the current level of adaptation and the difference between that level and the level of response required for the focal stimulus. Thus, the role of the standard in the COS effect is similar to the role of adaptation level-particularly in that both the relation between the target item and the adaptation level and the adaptation level itself are critical in both the encoding and decoding phases of the cycle. The emphasis on time- and context-dependent computation in our understanding of the COS effect is also similar to that emphasis in adaptation level theory. However, there are important differences between the COS effect and adaptation level theory. For example, the evidence suggests that the standard is at least somewhat cognitively accessible in the COS effect and that this standard is updated automatically not when new information is encountered, but only when it is used. However, it is not obvious that the adaptation level should be cognitively accessible, nor is it clear that the formation (or updating) of the adaptation level should take place only when required for use. Indeed, it seems that the perceptual system's response would be affected immediately on presentation of the information, suggesting immediate and automatic updating of the adaptation level. Thus, the extent to which the present findings extend to adaptation levels is an open empirical question.

\section{Combining Impression and Standard}

The influence of the impression on target recall is not a function of the context at encoding only. Previous research suggests that the effect of the impression on target recall is a function of the encoding context only because the effect of the impression on recall is estimated by the difference between target recall and the unbiased average. However, to accurately estimate the influence of the impression on recall, one must instead compare the target recall to the distorted standard actually used by the participants. We have shown that the influence of the impression on recall is small when the context changes between sessions but is larger when the context is consistent. Furthermore, these differences in the influence of the impression on recall appear to be due to an adjustment relating to participants' use of their impression of the target. However, this adjustment occurs only when there are tasks at Session 2 that require some integration across the two sessions. The presentation of new context items per se was not sufficient to change the use of the impression.

How did the participants adjust the use of their impressions when they (apparently) did not remember the target value? And why did they do so only when they had performed a task requiring integration of information across the two sessions? We propose that the participants engaged in plausible reasoning with respect to the target impression, allowing them to make the adjustment, even though they could not remember the target's specific values. There are several specific forms of such plausible reasoning that could account for change in the use of the impression at decoding. Most obviously, participants could have adjusted their original impressions. For example, in the $\mathrm{L}-\mathrm{H}$ condition at Session 1, participants formed the impression that the target company was very expensive; but after learning about expensive caterers in Session 2 and noticing the difference from Session 1, they may have reevaluated their original impression and adjusted it to be less extreme. Alternatively, instead of adjusting the extremity of their original impression, participants might have just been less confident in their impression. Thus, participants may have been less willing to use their impression at decoding, although they still thought of the target as being very expensive. A third possibility is that participants adjusted the relation between the scale on which the impression was represented and the scale of actual values (e.g., price). In this case, perhaps the participants still confidently believed that the target was very expensive but later believed that being very expensive implied less (or more) of an actual price difference from the average. Differentiating among these possibilities is a project for future research.

There are two important commonalities among these possibilities, however. In all cases, the adjustment depended on both knowing that the impression was relative and noticing that the context had changed (or had been maintained) across the two sessions. On the other hand, if a person did not explicitly realize either continuity or change in sentence lengths, they might have just used their original impression, with no adjustment at all. We return to this point below when we discuss the roles of understanding and awareness in the COS effect.

An interesting similarity exists between "metrics and mapping" research on numerical estimation (e.g., Brown $\&$ Siegler, 1993) and the COS effect, in terms of how metric information can affect mapping information. Mapping information is information that specifies relative values of the entities with respect to each other-for instance, knowing that the U.S. has a much higher population than Canada, without knowing either population value. Metric information is information that specifies aspects of the underlying quantitative property; for example, one might believe that the populations of countries range from over 1 billion down to about 1 million. People often have relatively good mapping information, but their metric information tends to be relatively impoverished (Brown \& Siegler, 1993). However, presentation of relatively little amounts of accurate metric information (a process called seeding) can, in combination with the participants' own mapping information, lead to much improved estimation. Particularly interesting for the present purposes, Brown and Siegler (Experiment 3) found that, when seed facts clearly implicated generalizations among categories of items, the seeds (metric information) could also affect the relative orderings of items (mapping information) rather than just affecting the overall accuracy of the estimations. For example, participants usually overestimated the populations of European countries and underestimated the populations of East Asian countries. However, presenting (as seeds) a small European country and a large East Asian country specifically had an impact on estimates of other countries from these regions: The mappings became more accurate. Having seeds that disconfirmed the (inaccurate) relationship in the initial mapping information tended to remove some of that initial inaccuracy. Similarly, seeds 
that confirmed the initial inaccuracy in the mapping information could make the estimates for the new countries even less accurate, since these seeds confirmed the preexisting overestimation of European countries' populations relative to those of the East Asian countries. The important point for the present purposes is that this change of the mapping information requires some degree of plausible reasoning based on newly available metric information and is thus at least broadly analogous to the adjustment of the impression in the present study. In particular, simply presenting a seed or set of seeds does not, in general, greatly affect the mappings, just as simply presenting the Session 2 items did not lead to any change in the initial impression. However, when the particular set of seeds relates to an existing generalization, the mappings could be affected. This might be similar to our finding that tasks that require integration across sessions can affect the use of the impression in recall.

It seems likely that such plausible reasoning could be common to many areas that involve relative encodingdecoding cycles, although it may not be easily seen in other experimental paradigms. In particular, it might be difficult to see in research paradigms that do not include the whole encoding-decoding cycle. Also, it is unlikely to hold for nonhuman participants, as in Thomas's (1993) peak-shift research in discrimination learning, although it would be very interesting to know whether the relative encoding is decoded in an equivalent manner across contexts in such research. If it is not decoded in this way, it would imply that there are mechanisms other than plausible reasoning that give rise to what we have called the adjustment of the use of the impression. However, in such research, it is very difficult to fully isolate the effect of the relative encoding from changes to the standard, so this question remains unanswered for the moment.

\section{Awareness Versus Understanding}

Awareness does seem to be a contributor to the COS effect in two ways. First, participants seem to be unaware that their standard is distorted by recency. This is not terribly surprising, since such recency effects may be a relatively automatic aspect of retrieval in the memory system; thus, participants may have little access or insight into them. Nevertheless, this distortion is a major contributor to distortion in target recall. Second, the change in the use of the impression only occurs if a task requires integration across the contexts, presumably drawing attention to the change or continuity in context. Otherwise, participants appear to use their original impressions equivalently across conditions. In general, the participants appear to be mostly unaware of the change in the context until they are given a task that requires integration across the contexts. Neither of these aspects of awareness, however, is specifically what Higgins and Liberman (1994) were interested in - namely, the awareness of the relativeness of the impression. In terms of this aspect of awareness specifically, it seems that Higgins and Liberman were correct: Participants do seem to be aware that their impressions are relative. Otherwise, it is hard to see why they would adjust the use of their impressions when the task calls attention to the change or continuity of context. On the other hand, it seems that Higgins and Liberman might not have been completely correct in terms of whether participants understand what that relativeness implies for recall (or estimation). They concluded that participants understood that their judgment of the target was made in relation to the standard available (Context 1) but failed to understand that, when using that impression, it needed to be decoded relative to the original standard that was available (Context 1), not to the current standard (combination of Contexts 1 and 2). However, the present results show that the participants adjusted their use of the impressions once they were made aware of the change or continuity of context. It is important that the adjustment in the use of the impression tends to decrease the overall distortion in recall of the target, relative to what would be expected without the adjustment. This suggests both awareness and at least some understanding of the relativeness of the impressions and how they should be used at decoding.

\section{Conclusion}

The present experiments were designed to investigate the cognitive mechanisms underlying the COS effectparticularly, the access or creation of the standard at decoding and how that standard is combined with the impression of the target at decoding. Our findings identify two new contributors to the COS effect. First, the standard at decoding is distorted toward recent items because it is computed when needed and is thus influenced by the accessibility of the items in memory. Second, the combination of the standard and the impression at decoding is affected by the change (or continuity) of the context across the two sessions, but only when participants engage in a task that requires the integration of information across the two sessions. It is important to note that this adjustment in the combination of impression and standard appears to operate in an appropriate way: The adjustment tends to result in less distorted recall of the target than would otherwise be expected. Such adjustment suggests that people are aware that their original impressions were relative and that they understand (at least to some degree) the implications of such relative impressions. Finally, we believe that plausible reasoning is the mechanism underlying the decoding of the impression with respect to the standard and that this is why the adjustment depends on a participant's engaging in a task that highlights change (or continuity) across the sessions. Such a plausible-reasoning mechanism is likely applicable to many of the relative encodingdecoding cycles in other areas of cognition, social cognition, and perception.

\section{AUTHOR NOTE}

This research was supported by NSERC Research Grant 250028 to the first author. The authors thank Rich Lange and Vaidehi Seth for their help with data collection. Correspondence concerning this article should be addressed to T. L. Spalding, Department of Psychology, P-217 Biological Sciences Building, University of Alberta, Edmonton, AB, T6G 2E9 Canada (e-mail: spalding@ualberta.ca). 


\section{REFERENCES}

Brown, N. R. (2002). Real-world estimation: Estimation modes and seeding effects. In B. H. Ross (Ed.), The psychology of learning and motivation: Advances in research and theory (Vol. 41, pp. 321-359). San Diego: Academic Press.

Brown, N. R., \& Siegler, R. S. (1993). Metrics and mappings: A framework for understanding real-world quantitative estimation. Psychological Review, 100, 511-534.

Choplin, J. M., \& Hummel, J. E. (2002). Magnitude comparisons distort mental representations of magnitude. Journal of Experimental Psychology: General, 131, 270-286.

Clark, L. F., Martin, L. L., \& Henry, S. M. (1993). Instantiation, interference, and the change of standard effect: Context functions in reconstructive memory. Journal of Personality \& Social Psychology, 64, 336-346.

Glanzer, M., \& Cunitz, A. R. (1966). Two storage mechanisms in free recall. Journal of Verbal Learning \& Verbal Behavior, 5, 351-360.

Helson, H. (1964). Adaptation-level theory: An experimental and systematic approach to behavior. New York: Harper \& Row.

Higgins, E. T., \& Liberman, A. (1994). Memory errors from a change of standard: A lack of awareness or of understanding? Cognitive Psychology, 27, 227-258.

Higgins, E. T., \& LuRIE, L. (1983). Context, categorization, and recall: The "change-of-standard" effect. Cognitive Psychology, 15, 525-547.

HigGins, E. T., \& STANGOR, C. (1988). A “change-of-standard" perspective on the relations among context, judgment, and memory. Journal of Personality \& Social Psychology, 54, 181-192.

MALMI, R. A., \& SAMSON, D. J. (1983). Intuitive averaging of categorized numerical stimuli. Journal of Verbal Learning \& Verbal Behavior, 22, 547-559.
MurPhy, G. L. (2002). The big book of concepts. Cambridge, MA: MIT Press.

Mussweiler, T. (2003). Comparison processes in social judgment: Mechanisms and consequences. Psychological Review, 110, 472489.

PARducci, A. (1965). Category judgment: A range-frequency model. Psychological Review, 72, 407-418.

SMith, E. R. (1998). Mental representation and memory. In D. T. Gilbert, S. T. Fiske, \& G. Lindzey (Eds.), The handbook of social psychology (Vol. 1, pp. 391-445). New York: McGraw-Hill.

Thomas, D. R. (1993). A model for adaptation-level effects on stimulus generalization. Psychological Review, 100, 658-673.

TVersky, A., \& Kahneman, D. (1974). Judgment under uncertainty: Heuristics and biases. Science, 185, 1124-1131.

\section{NOTE}

1. Higgins and Lurie (1983) did not specifically state that the standard used at decoding is accurate. However, they implied this assumption by predicting that participants would report values higher or lower than "the category standard that had been established across the two sessions" (p. 529), depending on their impression: If the standard is moderate (e.g., 15 years) and Jones is judged as harsh, then, according to Higgins and Lurie, participants will recall values greater than 15. In particular, if Higgins and Lurie had not assumed an accurate average, they could not have known whether the recalled values were higher or lower than the standard.

(Manuscript received March 24, 2009; revision accepted for publication December 24,2009 .) 